\title{
ppótessional directory
}

Space reserved for Certified Consulting Meteorologists only. For rates apply to:

Executive Director, AMERICAN METEOROLOGICAL SOCIETY, 45 Beacon St., Boston, Mass. 02108

A. H. GLENN AND ASSOCIATES

ALFRED H. GLENN

\section{Certified Consulting Meteorologist}

Consultants in Meteorology and Oceanography

Registered Engineers in Louisiana and Texas

New Orleans Lakefront Airport, New Orleans, La. 70126:

504-241-2222

3927 Essex Lane, Houston, Tex. 77027: 713-621-3500

Cable Address: GLENN, New Orleans

WEATHER SERVICES CORPORATION

JOHN E. WALLACE

PETER R. LEAVITT

JOHN P. MURPHY

Certified Consulting Meteorologists

World wide operational forecasts, statistical research, environmental meteorology, forensic meteorology and expert testimony 131 A Great Road, Bedford, Mass. 01730 617-275-8860

TWX 710-326-0799

LEO ALPERT, Ph.D.

Certified Consulting Meteorologist

4122 Cobblestone Place

Durham, North Carolina 27707

$919-489-6479$

WALLACE E. HOWELL, Sc.D.

Certified Consulting Meteorologist

RFD 3 Box 400

Golden, Colorado 80401

303-277-9125

VINCENT J. SCHAEFER, Sc.D.

Certified Consulting Meteorologist

Research Consultant

R. D. 3, Box 36, Schermerhorn Road

Schenectady, New York 12306

EXpress 3-8978

518-393-8978
MURRAY AND TRETTEL, INCORPORATED

JOHN R. MURRAY

DENNIS W. TRETTEL

Certified Consulting Meteorologists

Operational Forecasts, Environmental Studies, Forensic Meteorology, Research Programs.

414 West Frontage Road, Northfield, Illinois 60093 312-446-7800

WEATHER CORPORATION OF AMERICA JAMES B. HARRINGTON, Ph.D.

ARMAND R. IACCHEO

Certified Consulting Meteorologists

Operational Forecasts

Applied Research

611 Olive Street, St. Louis, Missouri 63101

Phone: (314) 421-4737

Cable: Weather, St. Louis

NORTH AMERICAN

WEATHER CONSULTANTS

ROBERT D. ELLIOTT

EINAR L. HOVIND

JOHN T. WALSER

Certified Consulting Meteorologists

Weather Modification, Environmental and Air Pollution

Field Studies, Weather and Marine Forecasting, Clima-

tology, and Applied Research.

805-967-1246

Teletype: $910-334-1181$

Santa Barbara Municipal Airport, Goleta, Calif. 93017

METEOROLOGY RESEARCH, INC.

N. L. HALLANGER, WILLIAM B. MORELAND

Certified Consulting Meteorologists

Industrial Meteorology - Air Pollution Surveys

Field Investigations - Instrumentation

P.O. Box 637

464 West Woodbury Rd.

Altadena, Calif. 91001

213-791-1901

THE WALTER A. BOHAN COMPANY

WALTER A. BOHAN, DIRECTOR

Certified Consulting Meteorologist

Programs in Satellite Meteorology

Applied Research and Visual Communications

2026 Oakton Street, Park Ridge, Illinois 60068

312-825-3677 


\section{protessional directiony}

Space reserved for Certified Consulting Meteorologists only. For rates apply to:

Executive Director, AMERICAN METEOROLOGICAL SOCIETY, 45 Beacon St., Boston, Mass. 02108

AEROMET, INC.

D. RAY BOOKER, PH.D., PRESIDENT

\section{Certified Consulting Meteorologist}

Weather Modification Environmental Consulting Instrumentation Systems Airborne Instruments

P.O. Box FF

Norman, Oklahoma 73069

405-329-2424

LOREN W. CROW

\section{Certified Consulting Meteorologist}

2422 South Downing Street

Denver, Colorado 80210

303-722-8665

or 756-3971

SIDNEY R. FRANK . . . GROUP

SIDNEY R. FRANK, PRESIDENT

Certified Consulting Meteorologist

General Consulting for Industry and Government

Environmental Research \& Development Programs

Pollution Analyses-Theoretical and Operational

1500 Cecil Cook Place

Santa Barbara Municipal Airport

805-964-4477

FAA Building

Goleta, Calif. 93017

Home: $805-965-7540$

$\mathrm{ClC}$

Colorado International Corporation of Delaware Certified Consulting Meteorologist

RALPH PAPANIA, JR.

Weather modification operations and evoluation studies-cloud physics research-radar meteorology-cloud modeling applications -airborne instrumentation-meteorology and air quality monitoring stations-upper air soundings and data analysis.

P. O. Box 3007, Boulder, Colorado 80303

(303) 443-0384

TWX: $910-940-3444$

INSTITUTE FOR STORM RESEARCH

at the University of St. Thomas

JOHN C. FREEMAN, DIRECTOR OF RESEARCH

JOSEPH L. GOLDMAN, ASSOCIATE DIRECTOR OF RESEARCH

\section{Certified Consulting Meteorologists}

Research in theoretical and applied meteorology, oceanography, mathematics

$4104 \mathrm{Mt}$. Vernon, Houston, Texas 77006

713-529-4891 Telex 762-771

\section{METRO MONITORING SERVICES}

ERWIN K. KAUPER

Certified Consulting Meteorologist

Environmental Impact and Site Surveys.

Air Pollution Field Studies. Diffusion Analysis.

Weather and Air Quality Forecasting.

Air Pollution Monitoring Instrumentation.

274 East Rowland Avenue, Covina, CA 91723

213-332-8411

\section{E. BREWSTER BUXTON}

\section{Certified Consulting Meteorologist}

AVIATION-Airport Weather Lab and Analysis Center

Box 322, Park Street

Morrisville, Vermont $05661 \quad$ Morrisville-Stowe Airport

802-888-4566

802-888-5150

\section{RICHARD E. CALE}

\section{Certified Consulting Meteorologist}

Environmental Research Applications

Aviation Weather Investigations

Planning Services

P.O. Box 366, Cerritos, California 90701

213-926-6149

R. ROBERT RAPP, Ph.D.

Certified Consulting Meteorologist

4752-B La Villa Marina

Marina del Rey, California 90291

213-823-8808

\section{W. BOYNTON BECKWITH}

\section{Certified Consulting Meteorologist}

111 So. Baybrook Dr., 413

Palatine, Illinois 60067

312-991-0609

(Office) 312-952-6301 
Space reserved for Certified Consulting Meteorologists only. For rates apply to:

Executive Director, AMERICAN METEOROLOGICAL SOCIETY, 45 Beacon St., Boston, Mass. 02108

AEROVIRONMENT INC.

PAUL B. MAC CREADY JR., PH.D., PRESIDENT

\section{Certified Consulting Meteorologist}

Air quality observations and projections-freeways, airports, industrial. Consulting; programs; instrumentation: air data systems, acoustic radar. Diffusion, tracing, modeling. Weather modification. Aerodynamics.

145 North Vista Avenue

Pasadena, California 91107

$213-449-4392$

\section{HALES AND CO.}

Environmental Consultants

J. VERN HALES, PH.D.

\section{Certified Consulting Meteorologist}

Air and Water Quality, Environmental Impact Studies,

Field Programs, Remote Sensing, Atmospheric Physics

66 Brookmead Rd., Wayne, Pa. 19087

215-687-2330

\section{BERNARD H. DeWITT \& ASSOCIATES}

Certified Consulting Meteorologist

Consultants in Meteorology \& Aviation Safety

Aviation-Industrial-Government
343 S. Main Street

Ann Arbor, Michigan 48108

313-769-4944
Floyd Pearson

528 Victoria Square

Brighton, Michigan 48116 313-227-5290

\section{TEMPO_GE'S CENTER FOR ADVANCED STUDIES \\ EDWARD J. TSCHUPP}

Certified Consulting Meteorologist

Environmental Research-Meteorology, Hydrology, Environmental Impact Statements, Technology Assessments, Model Development, Systems Analysis 777-14th Street N.W. Washington, D.C. 20005 Drawer QQ (202) $637-4500$

Santa Barbara, Cal. 93102 (805) 965-0551

GEORGE E. McVEHIL, Ph.D.

\section{Certified Consulting Meteorologist}

Industrial Meteorology, Air Pollution, Environmental Impact Analysis.

\section{TRC-THE RESEARCH CORPORATION} of New England

NORMAN E. BOWNE

GEORGE F. COLLINS

Certified Consulting Meteorologists

Environmental Scientists and Registered Professional EngineersApplied Research, Engineering, Consulting-Diffusion Meteorology \& Oceanography-Site Surveys-Environmental Impact ReportsLaboratory Services

125 Silas Deane Highway

Wethersfield, Conn. 06109

$203-563-1431$

C. EUGENE BUELL, Ph.D., P.E.

Certified Consulting Meteorologist

Kaman Sciences Corporation

1700 Garden of the Gods Road

Colorado Springs, Colo., 80907

303-473-5880

OSCAR TENENBAUM ASSOCIATES

OSCAR TENENBAUM, J. D.

Certified Consulting Meteorologist

Consultant and Research Services in MeteorologyClimatology-Hydrology-Statistics

Investigation and Expert Testimony in Aviation, Industry, Insurance, Law

39 Bellevue St., Newton, Mass. 02158

Residence: $617-332-7445$

Office: $617-244-0253$

ENVIRONMENTAL RESEARCH \&

TECHNOLOGY, INC.

DAVID B. SPIEGLER, MGR. AIRMAP OPERATIONS

Certified Consulting Meteorologist

- Air Monitoring Analysis and Prediction (AIRMAP(D) Systems;

- Total Environmental Impact Reports; - Diffusion Modeling and Analysis; Earth Resources Analysis and Planning

696 Virginia Road, Concord, Massachusetts 01742 (617-369-8910)

131-133 Eisenhower Lane, Lombard, Illinois 60148 (312-629-8740)

3955 Birch Street, Newport Beach, California 92660 (714-540-2564)

NORTHWEST ENVIRONMENTAL

TECHNOLOGY LABORATORIES, INC.

WILLIAM T. KREISS, Ph.D., PRESIDENT

\section{Certified Consulting Meteorologist}

Air, Water, Noise Monitoring and Modeling - Physical and Social Environmental Planning and Design - Environmental Impact Statements - Micro-Macro Meteorological and Climatological Studies - Weather Modification and Solar Energy Studies - Instrumentation - Applied Research

300-120th Avenue N.E., Bldg. Two, Suite 108

Bellevue, Washington 98005 (Seattle Area)

(206) $455-3570$ 


\section{professional directiory}

Space reserved for Certified Consulting Meteorologists only. For rates apply to:

Executive Director, AMERICAN METeOROlogiCAL SOCIETy, 45 Beacon St., Boston, Mass. 02108

APPLIED METEOROLOGY, INC.

W. ALAN BOWMAN

Certified Consulting Meteorologist

Consulting - Systems - Analysis

Suite 203

9000 Southwest Freeway

Houston, Texas 77036

(713) 777-0106
SIMPSON WEATHER ASSOCIATES

R. H. SMMPSON, PH.D., PRESIIENT

JOANNE smesox, PII.D., CHIEF sChentist

Certified Consulting Meteorologists

Consultants in-

- Tropical Meteorology

- Weather Modification

- Numerical Simulations

- Natural Hazard Assessments and Planning

- Environmental Statistics

804-295-6709

P.O. Drawer 5508

Charlotesville, Va. 22903 


\section{prótessional directiory}

Space reserved for Members only. For rates apply to:

Executive Director, AMERICAN METEOROLOGICAL SOCIETY, 45 Beacon St., Boston, Mass. 02108

\section{ALLEN WEATHER CORPORATION}

Meteorologists-Oceanographers

5207 Wisconsin Ave., N.W.

Washington, D.C. 20015

EMerson 3-7221

Cable: ALWEX WADC

NATIONAL WEATHER CORPORATION WORLD-WIDE WEATHER SERVICES

Forecasting-Flight Planning-Data Analysis-Surveys-Displays

Hangar Twelve, Newark Airport, Newark, N.J. 07114 201-624-8110

Orange County Airport

Santa Ana, Calif. 92707

213-776-5507

\section{Hangar D}

Westchester County Airport

White Plains, N.Y. 10601

$914-428-6500$

\section{METEOROLOGY INTERNATIONAL}

\section{INCORPORATED}

Technologies in the Analysis and Prediction of Physically and Operationally Significant Variabilities in the Atmosphere and Oceans, Satellite Meteorology, Circulation Dynamics, Computer Systems Applications

205 Montecito Avenue

Monterey, California 93940

408-372-5173

WEATHER TRENDS INTERNATIONAL, LTD.

Specializing in Long-Range Forecasts, Weather/Marketing Research \& Promotional Weather Services in North America and Europe

565 Fifth Ave., N.Y., N.Y. 10017 (212) MU 2-4280

European HQ: London

\section{GENERAL WEATHER CENTER}

Directed by Lyle E. Brosché.

Effective Long and Short Range Specialized Forecasts, and Consulting, for Business and Industry. Excellent Client References.

527 Fisher Building, Detroit, Michigan 48202

313-871-3511 TWX 810-221-1287

\section{DeNARDO \& McFARLAND}

Weather Services, Inc.

Municipal, Industrial and Aviation Forecasts; Atmospheric and Climatological Research Utilizing Computer Facilities

TWX 710-794-4804 412-462-6464

Allegheny County Airport, West Mifflin, Pennsylvania 15122

\section{ATMOSPHERICS INCORPORATED}

Operational Weather Modification and Cloud Physics Research Weather Radar and Meteorological Systems Applications Airborne and Ground Based Particulate Measurements

5652 E. Dayton Ave., Fresno, California 93727 209-291-5575

INTERNATIONAL WEATHER CORPORATION WALTER F. ZELTMANN, PRESIDENT

Consultant Meteorologists . . Oceanography

Forecasts ... Statistical Surveys ... Legal and Insurance

P. O. Box 176, Bay Ridge Station, Brooklyn, N. Y. 11220

212-854-0300

SOUTHEAST WEATHER CONSULTANTS, INC. Consultants For Research, Aviation \& Industry

AVMETS *

Specializing in Programming Jet Minimum Cost Cruise Control Flight Planning For Air Carriers and Business Jets

Southern Aero Building P.O. Box 20637

Atlanta Airport Branch, Atlanta, Georgia 30320

404-761-6991

- Aviation Meteorological Service

METRONICS ASSOCIATES, INC.

W. A. PERKINS, PRESIDENT P. A. LEIGHTON, BD. CHMN S. W. GRINNELL, L. M. VAUGHAN

Environmental Services ... Atmospheric Research

FP Air Tracer Systems . . Rotorod Samplers

Stanford Industrial Park

3201 Porter Drive

$415-493-5632$ 


\section{prótessional directory}

Space reserved for Members only. For rates apply to:

Executive Director, AMERICAN METEOROLOGICAL SOCIETY, 45 Beacon St., Boston, Mass. 02108

\section{WEATHER SURVEYS}

CONRAD B. GOSSET, Forensic Meteorologist

Specializing in investigations for

Law Firms and Insurance Companies

The County Trust Building, Pleasantville, N.Y. 10570

914-769-1350

\section{GULF COAST WEATHER SERVICE}

Commercial Section of

WTVT Television Weather Service

Serving individuals and industry since 1961

$\begin{array}{lr}\text { Roy Leep } & \text { Tampa, Florida } 33622 \\ \text { Director } & 813-876-3592\end{array}$

\section{ENVIRONMENTAL WEATHER SERVICE}

A Service of WFLA, Inc.

Legal - Insurance - Pollution Research - Aviation Industry Agricultural Forecast - Marine
P. O. Box 1410
Tampa, Florida 33601
Paul Catoe
813-229-7781
George Wooten
Patrick Moore

\section{GEOMET, INCORPORATED}

GEORGE H. MILLY, Ph.D., PRESIDENT

Air pollution analysis, modelling and monitoring; Environmental dato systems; weather modification studies; research and operations; instrumentation.

15 Firstfield Road

Gaithersburg, Maryland 20760

301-948-0755

2814-A Metropolitan Place Pomona, California 91767 714-593-1318

\section{LEONARD G. PARDUE}

Consulting Meteorologist

Florida Specialist Since 1948

- Weather Investigations for Law Firms

- Expert Testimony

- Staff Meteorologist, Radio WIOD

$305-888-5434$

641 Falcon Avenue Miami Springs, Florida 33166

BENDIX COMMERCIAL SERVICE CORP.

Marine Science Services

ROBERT A. RAGUSO, MANAGER

Optimum Ship Routing - Ocean Weather Forecasts - Marine Operations Consulting

P. O. Box 2205, South Hackensack, New Jersey 07606

$\begin{array}{lll}\text { (212) } 947-9000 & \text { or } 281) ~ 288-1550\end{array}$

TWX: 710-990-6114 Telex: 134-318 Cable: BEXMARSERV

\section{DAMES \& MOORE}

Consultants in the Environmental and Applied Earth Sciences

Services in Meteorology

Diffusion Studies - Regional Planning for Air Quality - Air Quality Monitoring - Atmospheric Heat Sink Studies - Nuclear Industry Safety Reports - Industrial Effects on Air Quality

Offices in 38 Principal Cities Throughout the World

CONTROL DATA CORPORATION

Meteorology Department, Research Division

Consulting Services

Assessment of air pollution potential; diffusion modelling - Design of air quality sampling and meteorological networks - Site survey - Climatological and statistical analyses - Mesoscale measurements with "METRAC" - Data processing systems

612-853-3595

P.O. Box 1249

Minneapolis, Minnesota 55440

ROBERT S. BUSH

Consulting Professional Meteorologist

7910 S.W. 52nd Avenue

Miami, Florida 33143

305-666-4406

\section{ACCU-WEATHER}

Weather Forecasting . . Climatological Data \& Surveys ... Specialized weather information ... Over a decade of service to business, government, the professions.

Joel N. Myers, Director

Elliot Abrams, Associate 814-237-0309 237-5801
622 West College Avenue State College, Pa. 16801 


\section{prótessional directiory}

Space reserved for Members only. For rates apply to:

Executrve Director, AMERICAN METEOROLOGICAL SOCIETY, 45 Beacon St., Boston, Mass. 02108

\section{CENTRAL WEATHER SERVICE}

over a quarter of a century of experience

EARL S. FINCKLE, PRESIDENT

Consulting Meteorologists

Radio Weathercasting Experts, Commodity Weather

Specialists

Aviation, Business and Industry, Government, Forensic Meteorology

Executive Office Building, Pal Waukee Airport

Wheeling, Illinois 60090

312-537-5920 TWX 910-651-2091

\section{MIDWEST WEATHER SERVICE}

Div. Commodity News Services, Inc.

Specializing in operational forecasts and consulting to industry, government media, aviation and agriculture; legal investigations and expert testimony.

1600 Genesee Street

Kansas City, Mo. 64102

816-471-0212

LOCKHEED ELECTRONICS COMPANY/

AEROSPACE SYSTEMS DIVISION

GERALD L. BARGER, PH.D.

JACK F. PARIS, PH.D.

DALE E. PHINNEY, M.S

Consulting Professional Meteorologists

Applied Research in - Statistical Climatology - Environmental Impact - Remote Sensing - Weather-Crop Modeling - Planning and Design Assistance

16811 El Camino Real, Houston, Texas 77058

Dr. Ken Bentley, Director, Science Applications-713-488-0080

\section{BALL BROTHERS RESEARCH CORP.}

Air Quality and Meteorological Consulting and Monitoring

Services, Environmental Data Collection Systems.

P. O. Box 1062, Boulder, Colorado 80302

Telephone 303-441-4592

\section{SIMPSON WEATHER ASSOCIATES}

ROGER PIELKE, PH.D.

RONALD BIONDINI, PH.D.
Consultants in-

- Tropical Meteorology

- Weather Modification

- Numerical Simulations
- Natural Hazard Assessments and Planning

- Environmental Statistics
804-295-6709 P.O. Drawer 5508 Charlottesville, Vo. 22903

\section{STEARNS-ROGER \\ COMPLETE ENVIRONMENTAL SERVICES}

Environmental impact statements . . Pollutant emission, air quality \& water quality monitoring . . . Dispersion estimates ... Ecological consulting ... . Meteorological field studies \& consulting services. Contact

ENVIRONMENTAL SCIENCES DIVISION

(303) 758-1122

P.O. Box 5888, Denver, Colorado 80217

C. W. THORNTHWAITE ASSOCIATES

WILLIAM J. SUPERIOR, PRESIDENT

Research and Field Studies

Sensors and Systems

Publications in Climatology

Route 1, Centerton

Elmer, New Jersey 08318

609-358-2350

METEOROLOGY COMPUTER SOFTWARE

JOSEPH A. CATALANO, Consultant

METEOROLOGY AND DATA PROCESSING

Numerical modelling

IMS data base management

Software packages for the Atmospheric Sciences

Time sharing access to air pollution models on latest computers

P.O. Box 7505

213-790-7202

WESTINGHOUSE ENVIRONMENTAL SYSTEMS DEPARTMENT METEOROLOGY AND AIR QUALITY STUDIES AND SERVICES

Field studies, air quality assessment, diffusion modeling and analysis, supplemental emission control programs and analysis, ground plume tracking.

Monroeville Mall, Monroeville, PA 15146

412-256-5640

\section{YORK RESEARCH CORPORATION}

Air Pollution Meteorology, Diffusion Modeling \& Analysis, Environmental Impact Statements, Meteorological \& Air Quality Monitoring Networks, Plant Siting Studies, Upper Air Studies, Expert Testimony, Complete Analytical Laboratory

DEPARTMENT OF ATMOSPHERIC SCIENCES

Edward J. Kaplin

One Research Drive

Director 203-325-1371 
Space reserved for Members only. For rates apply to:

Executive Director, AMERiCAN METEOROLOGICAL SOCIETY, 45 Beacon St., Boston, Mass. 02108

\title{
GEO-ATMOSPHERICS CORPORATION
}

\author{
WAYNe D. MOUNT, Ph.D., PRESIDENT
}

- Analysis, Design \& Engineering of Automated Meteorological Data Acquisition, Processing, \& Dissemination Systems - Worldwide Climatological Studies for Planning \& Design • Field Studies - Air \& Water Quality Assessments - Transport \& Diffusion Modeling - Performance Analyses of Intermittant Control Strategies • Environmental Impact Assessments \& Statements • Business Economics, Marketing \& New Ventures

P.O. Box 177, Lincoln, Mass. 01773 (617) 259-9685 